\title{
Recurrent Negative Pressure Pulmonary Edema
}

\author{
Vikas Pathak, MD; Iliana S. Hurtado Rendon, MD; \\ and Ronald L. Ciubotaru, MD
}

\begin{abstract}
An African-American man, aged 34 years, underwent an elective uncomplicated right wrist laceration repair while under general anesthesia. Following extubation, the patient developed hypoxemia, tachypnea, shortness of breath, pulmonary rales, frothy sputum, decreased oxygen saturation, and evidence of upper airway obstruction. Chest radiograph showed pulmonary edema. The patient was diagnosed with post-extubation pulmonary edema (aka. negative pressure pulmonary edema [NPPE]) and was treated with intravenous furosemide and oxygen therapy; he improved remarkably within a few hours. Once stabilized, the patient described a similar episode 10 years earlier following surgery for multiple gunshot wounds.

Negative pressure pulmonary edema following tracheal extubation is an uncommon (0.1\%) and life-threatening complication of patients undergoing endotracheal intubation and general anesthesia for surgical procedures. The common pattern in these cases is the occurrence of an episode of airway obstruction upon emergence from general anesthesia, usually caused by laryngospasm. Patients who are predisposed to airway obstruction may have an increased risk of airway complications upon extubation after general anesthesia. Prevention and early relief of upper airway obstruction should decrease incidence.
\end{abstract}

Recurrent NPPE has not been previously described in the literature. Herein, we describe the first case of recurrent NPPE in the same patient following extubation.

Keywords: Negative pressure pulmonary edema; Recurrence; Anesthesia

Reprint Requests:

Vikas Pathak, MD

Department of General Internal Medicine

Marshfield Clinic

1000 North Oak Avenue

Marshfield, WI 54449 USA

Tel: $7|5-221-7| 77$

Fax: 715-389-5757

E-mail: drvikaspathak@gmail.com

Received: April 18, 2010

Revised: July 21, 2010

Accepted: July 28, 2010

doi: $10.3121 / \mathrm{cmr} .2010 .936$
$\mathrm{N}$ is an uncom pos (0.1\% prevalence) and life-threatening complication for patients is an uncommon ( $0.1 \%$ prevalence) and life-threatening complication for patients undergoing endotracheal intubation with general anesthesia for surgical procedures. Negative pressure pulmonary edema is also known as "postextubation pulmonary edema," "post-obstructive pulmonary edema," or "laryngospasm-induced pulmonary edema." The common pattern in these cases is the occurrence of an episode of airway obstruction upon emergence from general anesthesia, usually caused by laryngospasm. ${ }^{1}$

Upper airway obstruction secondary to glottis closure following extubation leads to marked increase in negative intrathoracic pressure. This causes pulmonary edema $^{2}$ which may be unilateral or bilateral. ${ }^{3,4}$

We herein describe the first reported case of recurrent negative pressure pulmonary edema in the same patient following extubation. 
Acute coronary syndrome was ruled out with serial cardiac enzymes and electrocardiogram (EKG). Echocardiogram showed normal ejection fraction with normal valves and no wall motion abnormality.

The patient was diagnosed with post-extubation pulmonary edema and was treated with intravenous furosemide and oxygen therapy. The patient improved remarkably within the next few hours and did not require reintubation.

Once stable, the patient mentioned that he had a similar episode 10 years prior. At that time, he had been admitted to one of the city hospitals in New York where he had undergone surgery for multiple gunshot wounds. During that admission, he had developed shortness of breath and hemoptysis after

Figure 1. Chest $X$-ray showing bilateral infiltrates, right greater than left, suggestive of pulmonary edema: The film was taken immediately after the patient became symptomatic.

\section{Case Presentation}

An African-American man, aged 34 years, underwent an elective uncomplicated right wrist laceration repair under general anesthesia. His medical history was significant for hypertension and hyperthyroidism, which were well controlled with hydrochlorothiazide and methimazole, respectively. Physical examination before surgery was unremarkable except for signs and symptoms related to the right wrist laceration. Laboratory values including TSH level were within normal limits. Admission chest X-ray was normal. The tracheal intubation, anesthesia, mechanical ventilation, and surgical procedure were uneventful.

Post-procedure the patient was extubated and sent to the postanesthesia care unit (PACU). Immediately upon arrival to the PACU, he became restless and developed marked shortness of breath. This was followed by frank hemoptysis and decreased arterial oxygen saturation. Stridor was absent, and there were no signs of airway obstruction. On examination the patient appeared in marked respiratory distress with respiratory rate of 30 breaths per minute, pulse 110 beats per minute, blood pressure $150 / 70 \mathrm{~mm} \mathrm{Hg}$, and temperature $98.2^{\circ} \mathrm{F}$. Oxygen saturation was $60 \%$ on $100 \%$ non-rebreather mask. On further examination, the jugular veins were found to be flat. Heart sounds were normal without any gallop or $4^{\text {th }}$ heart sound. Bilateral end-inspiratory crepitations were heard anteriorly and posteriorly (right $>$ left). The patient was awake and was transferred to the medical intensive care unit (ICU) for further management.

The patient was kept in a propped up position and treated with intravenous furosemide and oxygen. The arterial blood gases on $\mathrm{FIO}_{2}$ of $100 \%$ revealed $\mathrm{pH} 7.37, \mathrm{PCO}_{2} 44.5 \mathrm{mmHg}$, and $\mathrm{PO}_{2}$ $99.6 \mathrm{mmHg}$. Chest X-ray showed diffuse bilateral infiltrates, extubation and was treated in the ICU with "water pills" and oxygen therapy. His history was consistent with the medical records obtained from that hospital, and he had indeed been treated for NPPE post-extubation. Physical examination and laboratory values were within normal limits during that admission.

On the second day of admission in the ICU, the patient was comfortable with normal vital signs. Repeat chest X-ray showed marked resolution of the pulmonary infiltrates (figure 2). The patient was discharged home and advised to follow up in the medicine and orthopedics clinic in one week.

\section{Discussion}

Negative-pressure pulmonary edema (NPPE) is a noncardiogenic pulmonary edema in which a large, negative intrathoracic pressure generated against an obstructed upper airway results in the shift of fluid into the interstitium of the lungs.

The prevalence of NPPE is reported to be $<0.1 \%{ }^{1}$ and usually involves young healthy patients. ${ }^{5-7}$ But we were unable to find any cases of recurrent NPPE reported in the current literature.

During episodes of upper airway obstruction, inspiratory efforts against a closed glottis (modified Mueller maneuver) may result in markedly negative pleural pressures (greater than $-100 \mathrm{cmH}_{2} \mathrm{O}$ ). The resultant decreased intrathoracic pressure leads to increased venous return to the right side of the heart and increased hydrostatic pulmonary capillary pressure. In addition, altered pulmonary vascular resistance by hypoxia and increased adrenergic state may result in dilation of the right ventricle, interventricular septum shift to the left, and left ventricular diastolic dysfunction. Thus, the combination of increased venous return and increased pulmonary capillary wedge pressure favors the shift of fluid into the pulmonary interstitium causing pulmonary edema. 


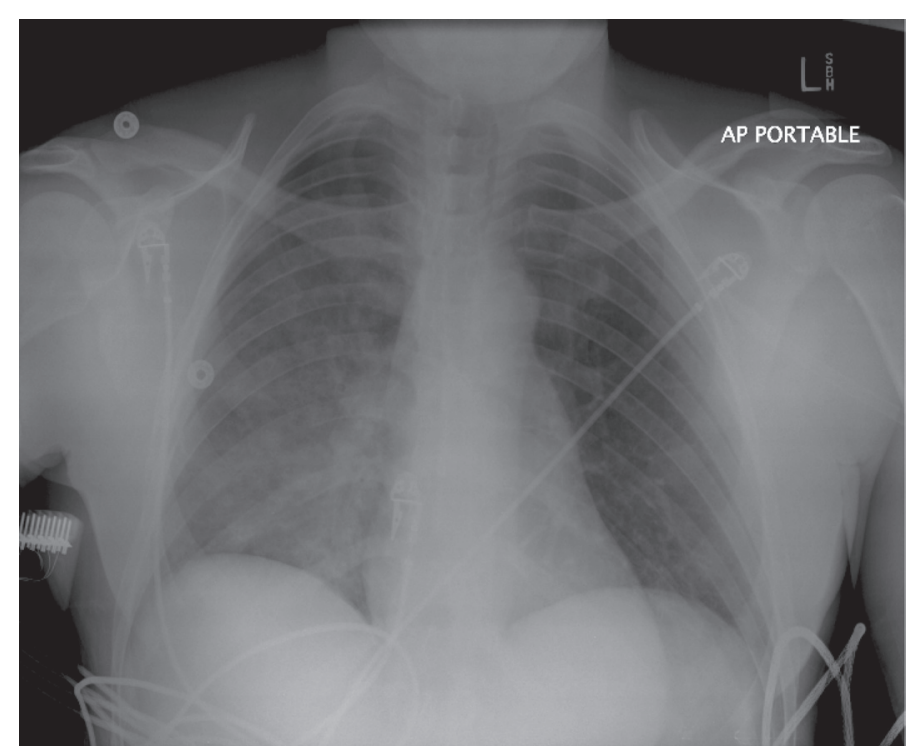

Figure 2. Chest X-ray taken on $2^{\text {nd }}$ day of admission in the ICU showing marked resolution of the pulmonary edema.

Patients who are predisposed to airway obstruction may have an increased risk of airway complications upon extubation after general anesthesia. ${ }^{8}$ The risk factors associated with airway obstruction are obesity, palatal mass, short thick neck, obstructive sleep apnea syndrome, and post-nasal surgery. ${ }^{8-12}$ Our patient did not have any of the above mentioned risk factors.

Signs and symptoms of NPPE include hypoxemia, tachypnea, shortness of breath, pulmonary rales, frothy sputum production, decreased oxygen saturation, and evidence of upper airway obstruction. Chest radiographs may show pulmonary edema. Hemoptysis ${ }^{13-15}$ is another symptom that may be present, which in the setting of NPPE signifies capillary failure and alveolar hemorrhage. ${ }^{16-18}$ Our patient presented with all these above symptoms on both occasions after he was extubated.

Other causes of pulmonary edema such as aspiration of gastric contents, acute respiratory distress syndrome (ARDS), congestive heart failure (CHF), volume overload, and pulmonary embolism should be kept in mind while working up these patients. Given that our patient was a relatively healthy young patient who developed pulmonary edema immediately after extubation, and all other tests including echocardiogram, EKG and cardiac enzymes were negative, we effectively ruled out all the above-mentioned causes of pulmonary edema.

As in our case, most patients who develop NPPE do well with only oxygen and furosemide, and few require monitoring in the ICU, although rarely some patients do require intubation. The aim of treatment is to relieve laryngospasm, maintain airway patency, and ensure adequate oxygenation. Positive pressure ventilation with a bag-valve mask and paralytics are often used to accomplish this goal. If manual ventilation is ineffective, short acting muscle relaxants such as succinylcholine can be used to relieve laryngospasm. In some instances, reintubation and mechanical ventilation are required for a short duration to maintain airway patency and adequate oxygenation.

The role of furosemide is controversial, since some authors believe that NPPE is secondary to leaky capillaries and not to excess fluid or fluid overload. ${ }^{1,19}$ But in our patient, furosemide was beneficial with a good symptomatic and radiological outcome.

As in our case, patients with NPPE recover well, with rapid and complete resolution of both clinical and radiological features within 24 hours, and there is no sequelae once recovery has occurred. The good pre-existing health condition of many patients who develop NPPE may explain the rapid resolution of this condition. ${ }^{20}$

Prevention and early relief of upper airway obstruction should decrease the incidence. Preventive measures such as use of an intra-operative muscle relaxant, topical or spray lidocaine, and steroids should be used. Although it is not common to use steroids, ${ }^{21}$ they have been used in patients who are at risk.

Other preventive measures that have been suggested are to clear the airway of the retained blood or secretions by suctioning the oropharynx and extubating the patient in either a very deep or very light level of anesthesia, when the risk of laryngospasm is not as great. Prophylactic continuous positive airway pressure (CPAP) has also been advocated in at-risk patients. ${ }^{22}$

\section{Conclusion}

Recurrent NPPE in the same patient is a life threatening condition that has not been previously documented in the literature. This is probably because NPPE itself is an uncommon occurrence, and it is very difficult to find instances of recurrent NPPE. Also, physicians may not normally ask about it while doing pre-anesthetic evaluations.

Recurrent NPPE should be kept in mind while administering general anesthesia in a young patient, and the patient should be specifically asked whether a history of any such occurrence exists. Any positive past history should prompt the physician to take prophylactic measures to prevent recurrent NPPE and decrease the associated morbidity. Early recognition allows prompt application of positive airway pressure and a rapid resolution.

\section{References}

1. Deepika K, Kenaan CA, Barrocas AM, Fonseca JJ, Bikazi GB. Negative pressure pulmonary edema after acute upper airway obstruction. J Clin Anesth 1997;9:403-408.

2. Willms D, Shure D. Pulmonary edema due to upper airway obstruction in adults. Chest 1988; 94:1090-1092. 
3. Goodman BT, Richardson MG. Case Report: unilateral negative pressure pulmonary edema- a complication of endobronchial intubation. Can J Anaesth 2008:55:691-695.

4. Bourke AM. Unilateral pulmonary oedema following post extubation laryngospasm. Anaesthesia 1997;52:928.

5. Anderson AF, Alfrey D, Lipscomb AB Jr. Acute pulmonary edema, an unusual complication following arthroscopy: a report of three cases. Arthroscopy 1990;6:235-237.

6. Holmes JR, Hensinger RN, Wojtys EW. Postoperative pulmonary edema in young, athletic adults. Am J Sports Med 1991;19:365-371.

7. Goitz RJ, Goitz HT, DiFazio CA, McCue FC 3rd. Identification of acute pulmonary edema following routine outpatient orthopedic procedures in healthy, young athletes. Orthopedics 1994; 17:949-951.

8. Lorch DG, Sahn SA.: Post-extubation pulmonary edema following anesthesia induced by upper airway obstruction: Are certain patients at increased risk? Chest 1986; 90:802-805.

9. Kamal RS, Agha S. Acute pulmonary oedema. Anaesthesia 1984;39:464-467.

10. Cozanitis DA, Leijala M, Pesonen E, Zaki HA. Acute pulmonary oedema due to laryngeal spasm. Anaesthesia 1982;37:1198-1199.

11. Jackson FN, Rowland V, Corssen G. Laryngospasm-induced pulmonary edema. Chest 1980;78:819-821.

12. Melnick BM. Postlaryngospasm pulmonary edema in adults. Anesthesiology 1984;60:517-518.

13. Bhavani-Shankar K, Hart NS, Mushlin PS. Negative pressure induced airway and pulmonary injury. Can J Anaesth 1997;44:78-81.

14. Koch SM, Abramson DC, Ford M, Peterson D, Katz J. Bronchoscopic finding in post-obstructive pulmonary oedema. Can J Anaesth 1996;43:73-76.

15. West JB, Mathieu-Costello O. Stress failure of pulmonary capillaries: role in lung and heart disease. Lancet 1992;340:762-767.

16. Schwartz DR, Maroo A, Malhotra A, Kesselman H. Negative pressure pulmonary hemorrhage. Chest 1999;115:1194-1197.

17. Broccard AF, Liaudet L, Aubert JD, Schnyder P, Schaller MD. Negative pressure post-tracheal extubation alveolar hemorrhage. Anesth Analg 2001;92:273-275.

18. Patel AR, Bersten AD. Pulmonary haemorrhage associated with negative-pressure pulmonary oedema: a case report. Crit Care Resusc 2006;8:115-116.

19. Sulek CR, Kirby RR. The recurring problem of negativepressure pulmonary edema. Curr Rev Clin Anesth 1998;18:241-252.

20. Nishizawa M, Chaffee TL, Goto H. Pulmonary edema induced by endotracheal tube obstruction: a case report. Respir Care 1993;38:479-481.

21. Bagshaw SM, Delaney A, Farrell C, Drummond J, Brindley PG. Best evidence in critical care medicine. Steroids to prevent post-extubation airway obstruction in adult critically ill patients. Can J Anaesth 2008 ;55:382-385.

22. Galvis AG, Stool JE, Bluestone CD. Pulmonary edema following relief of upper airway obstruction. Ann Otol Rhinol Laryngol 1980;89:124-128.

\section{Author Affiliations}

Vikas Pathak, MD*; Iliana S. Hurtado Rendon, $M D^{* *}$; Ronald L. Ciubotaru, $M D^{*}$

${ }^{*}$ Department of Internal Medicine, St. Barnabas Hospital, Bronx, NY USA

†Pulmonologist/Intensivist, Director of ICU, St. Barnabas Hospital, Bronx, NY USA

†Current affiliation: Department of Internal Medicine, Marshfield Clinic, Marshfield, WI USA 